\title{
Simplifying the Numerical Human Model with $k$-means Clustering Method
}

\author{
Kyoko Fujimoto, Leonardo M. Angelone, Sunder S. Rajan, \\ and Maria Ida Iacono
}

\section{Introduction}

Computational modeling is widely used to assure patient safety with respect to radiofrequency (RF) related concerns during magnetic resonance imaging (MRI). It allows for evaluation of RF power absorption and specific absorption rate (SAR) in anatomically detailed numerical human models. Such evaluations are especially important for safety of patients with implantable devices.

RF-induced heating depends on the physical characteristics of the patient [1], and as such, it is expected that numerical human models are sufficiently detailed to be able to estimate the differences across the patient popupation; however, the timeconsuming model generation process prevents achieving a realistic safety evaluation.

A limited number of anatomically realistic numerical human models are available for research and development use. Currently available whole-body numerical models have 26-77 anatomical structures [2-5]. A previous study showed that three different dielectric properties (muscle, fat, and lung) were sufficient to estimate SAR with a 5-mm resolution [6]; yet there are implantable devices with dimensions of less than $5 \mathrm{~mm}$ that can be implanted in very thin anatomical structures such as arteries and veins. Thus, a more detailed model may be needed for robust RF-induced heating evaluation. Moreover, a study using a higher resolution model showed that the blood vessel SAR can be up to ten times higher than the maximum standard gel phantom SAR value [7]. Knowing the limits of simplification in a numerical model can help not only to reduce the time needed for segmentation for

K. Fujimoto $(\bowtie) \cdot$ L. M. Angelone $\cdot$ S. S. Rajan · M. I. Iacono

Center for Devices and Radiological Health, US Food and Drug Administration, Silver Spring, MD, USA

e-mail: kyoko.fujimoto@fda.hhs.gov 
model generation but also to fabricate a standard phantom which can accurately reflect the in vivo result.

In this study, we used the $k$-means clustering method, one of the commonly used vector quantitization methods for cluster analysis, to reduce the number of anatomical structure types with different dielectric properties in a detailed human model. Then we investigated the resulting differences in SAR with respect to the number of clusters. The simplified models were then used to simulate a test senario for RF-exposure by calculating background tangential electric field $\left(\mathrm{E}_{\mathrm{tan}}\right)$ along five stent trajectories in selected arteries.

\section{Methods}

\section{1 k-means Clustering}

The $k$-means clustering was applied on the electrical conductivity and permittivity of anatomical structures of the AustinMan model [5] with 61 different anatomical structures in MATLAB (The MathWorks Inc., Natick, MA, United States). Ten model variations with different dielectric property configurations were used in the simulations: one full model with the original 61 anatomical structures with 51 dielectric properties and nine models with varying number of dielectric property clusters $(k=33,30,27,24,21,18,15,12$, and 9). The total sum of distances was used as a distance measure, and the model with 33 clusters was chosen as a starting point because the distance was less than 0.5 for numbers of clusters above 34 . The example of clustering for $k=9$ is shown in Fig. 1(a).

\subsection{Computational Modeling Setups}

The computational modeling setups were implemented using the commercially available finite-difference time-domain platform Sim4Life (Zurich Med Tech, Switzerland). A 32-port 16-rung birdcage coil, $700 \mathrm{~mm}$ in length and $650 \mathrm{~mm}$ in diameter, with idealized excitation was modeled. All the current carrying coil structures were modeled as perfect electric conductors (PEC). Electromagnetic simulations were performed by feeding the coil with a continuous sinusoidal wave at $128 \mathrm{MHz}$.

The Huygens' approach [8] was applied to facilitate a fair comparison between full and simplified models. The incident field was calculated with an unloaded coil first, then used to compute electromagnetic fields within body models, with 1-mm isotropic grid. The modeling software could estimate electomagnetic fields with a coarser grid, especially with the clustered models, which would reduce computational burdens. However, to facilitate fair comparisons between the full model and 


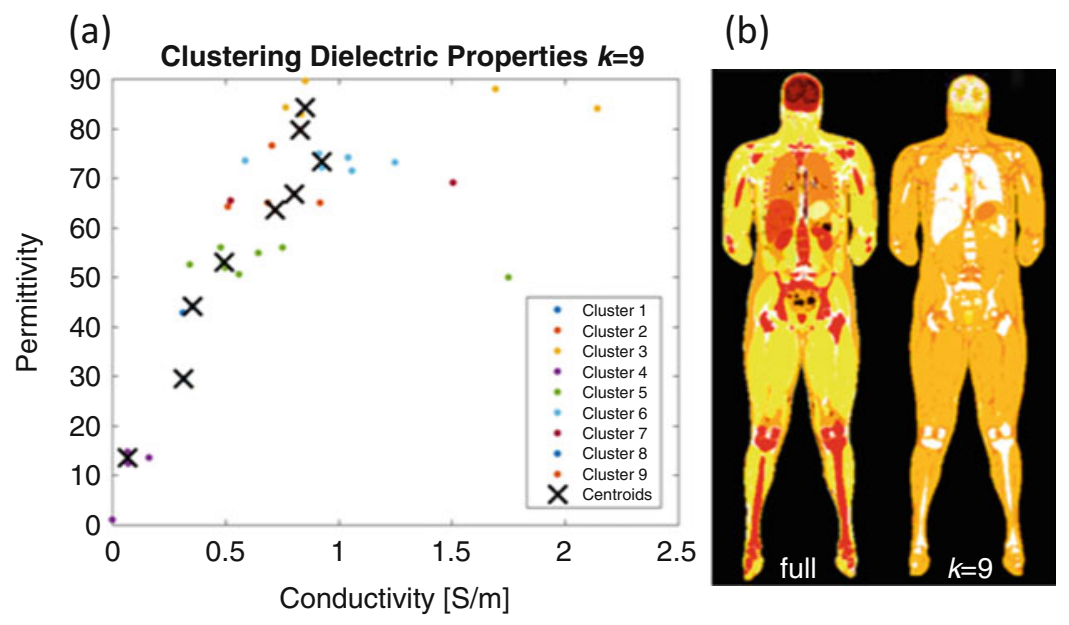

Fig. 1 (a) Example of the $k$-means clustering based on the sum of absolute differences. Different colors represent different clusters. Calculated centroid values were used in the simulation for anatomical structures within clusters. (b) The coronal view of the segmented model with full properties and nine properties is shown

the clustered models, the same resolution of the AustinMan model was used to discretize the models in this study. All the models were simulated at the hip bone imaging landmark.

\subsection{SAR Calculation}

The single-voxel SAR $\left(\mathrm{SAR}_{\text {raw }}\right), 1$ g-averaged SAR $\left(\mathrm{SAR}_{1 \mathrm{~g}}\right)$, and 10 g-averaged SAR $\left(\mathrm{SAR}_{10 \mathrm{~g}}\right)$ results were compared by calculating the mean and maximum percentage difference between the full model and the clustered models. Voxelwise comparison of each pair was performed by linear regression of the SAR values. All SAR values were computed with original mass density values of the model and normalized to a whole-body averaged SAR equal to $2 \mathrm{~W} / \mathrm{kg}$ [9]. All the analyses were performed in MATLAB.

\subsection{Electric Field Tangential to Stents in Blood Vessels}

To simulate a test case of RF heating assessment, stent trajectories were chosen in the five locations in the arteries of AustinMan as described in Fujimoto et al. [10]. Five case studies were analyzed for the ascending aorta, the brachial, the 
femoral, the iliac, and the popliteal arteries. Stent trajectories were created based on the centerline of each blood vessel that was calculated in MATLAB by binarizing a selected vessel and determining the centroid of the consecutive axial slices of the model. The centerline was then imported into Sim4Life to create a smooth trajectory. The $E_{\tan }$ value was calculated along each trajectory using the IMSAFE module in Sim4Life. The magnitudes of $E_{\text {tan }}$ values were calculated offline for each number of clusters.

\section{Results}

The values of dielectric properties for each $k$-clustered model were determined by the $k$ centroids. The example of clustering plot and coronal slices of full and $k=9$ models are shown in Fig. 1. The centroids as shown in Fig. 1(a) are distributed across the range of the original permittivity values from 1 (air) and 90 (kidney) and the original conductivity values from 0 (air) to 2.1 (cerebrospinal fluid). For example, with these centroids, skin, muscle, diaphragm, and liver became one cluster in the $k=9$ clustered model. The maximum intensity projection of each SAR map showed that the $\mathrm{SAR}_{1 \mathrm{~g}}$ and $\mathrm{SAR}_{10 \mathrm{~g}}$ maps were qualitatively similar among different models regardless of numbers of dielectric properties used (Fig. 2).

The mean and maximum percentage difference (Table 1) revealed that there were up to $15.3 \%$ mean difference in SAR. The example cross-sectional (transverse slice) SAR maps are shown in Fig. 3. The 12-clustered models estimated higher $\mathrm{SAR}_{\text {raw }}$ values compared to the full model on the skin, whereas the values for $\mathrm{SAR}_{1 \mathrm{~g}}$ and $\mathrm{SAR}_{10 \mathrm{~g}}$ were similar. This trend was observed when compared between the rest of the simplified models and the full model. The SAR values from the full model plotted against the SAR values from each clustered model revealed that all the clustered models showed high correlations with the full model (Fig. 4). The $\mathrm{E}_{\mathrm{tan}}$ values calculated in selected stent paths were similar among the full and clustered models (Fig. 5).

\section{Discussion}

The clustered analysis showed that reducing the number of dielectric properties from 51 (original) to 30 has less than $0.2 \%$ effect on the mean SAR results. Further reduction in the number of dielectric properties was not linearly correlated with mean and maximum SAR differences. The greatest mean SAR difference was $15.3 \%$ for $k=18$. Each voxel pair between the full and the 30-clustered SAR values was highly correlated. Our results suggest not only reducing the segmentation time on 
Fig. 2 Maximum intensity projection of $\mathrm{SAR}_{1 \mathrm{~g}}$ and $\mathrm{SAR}_{10 \mathrm{~g}}$ from the simulations with the models with full, 33-clustered, and nine-clustered properties. No subjective differences were observed among the three $\mathrm{SAR}_{1 \mathrm{~g}}$ and $\mathrm{SAR}_{10 \mathrm{~g}}$ maps
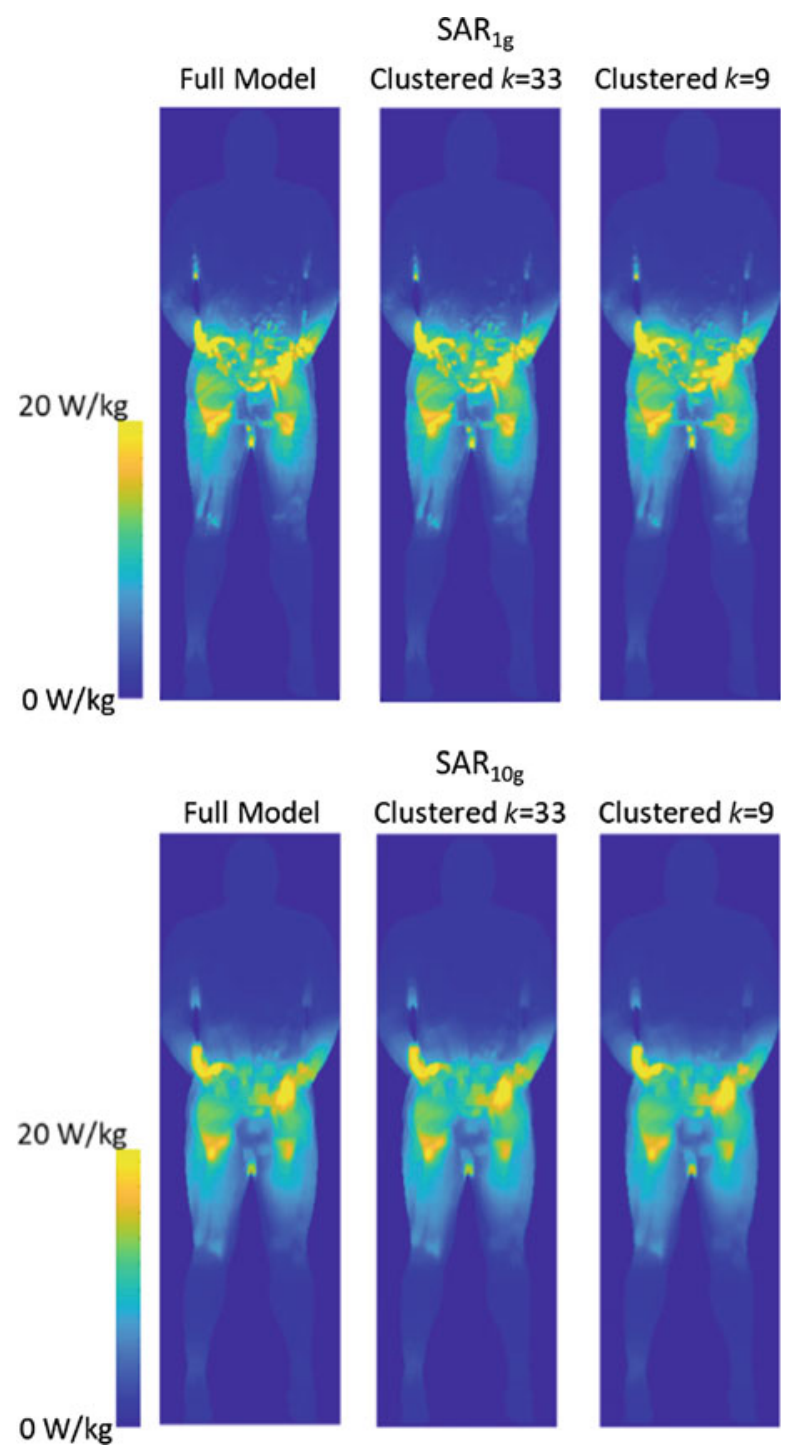

generating models but also using existing models with less anatomical structures which result in reduction in computational time.

The $\mathrm{E}_{\mathrm{tan}}$ results (Fig. 5) showed that they were not linearly correlated with the number of clusters. In other words, as shown with the ascending aorta, the iliac, and the femoral artery trajectories, the models with small numbers of clusters can estimate the $\mathrm{E}_{\mathrm{tan}}$ results as the full model simulation does. 
Table 1 Percent differences for mean and maximum values computed between full and clustered $\mathrm{SAR}_{\text {raw }}, \mathrm{SAR}_{1 \mathrm{~g}}$, and $\mathrm{SAR}_{10 \mathrm{~g}}$ maps

\begin{tabular}{|c|c|c|c|c|c|c|}
\hline \multicolumn{7}{|c|}{ Percent difference between full and clustered SAR maps } \\
\hline \multirow[b]{2}{*}{ \# Clusters } & \multicolumn{2}{|c|}{$\mathrm{SAR}_{\text {raw }}$} & \multicolumn{2}{|l|}{$\mathrm{SAR}_{1 \mathrm{~g}}$} & \multicolumn{2}{|c|}{$\mathrm{SAR}_{10 \mathrm{~g}}$} \\
\hline & Mean & Maximum & Mean & Maximum & Mean & Maximum \\
\hline 33 & $0.0 \%$ & $54.9 \%$ & $0.0 \%$ & $114.8 \%$ & $0.0 \%$ & $152.7 \%$ \\
\hline 30 & $0.2 \%$ & $56.9 \%$ & $0.2 \%$ & $114.8 \%$ & $0.1 \%$ & $152.7 \%$ \\
\hline 27 & $3.0 \%$ & $177.8 \%$ & $2.8 \%$ & $115.0 \%$ & $2.3 \%$ & $152.6 \%$ \\
\hline 24 & $2.7 \%$ & $53.8 \%$ & $2.5 \%$ & $115.1 \%$ & $2.1 \%$ & $152.6 \%$ \\
\hline 21 & $15.1 \%$ & $151.8 \%$ & $11.7 \%$ & $117.4 \%$ & $9.4 \%$ & $156.3 \%$ \\
\hline 18 & $15.3 \%$ & $168.9 \%$ & $11.8 \%$ & $116.5 \%$ & $9.3 \%$ & $156.6 \%$ \\
\hline 15 & $8.1 \%$ & $171.8 \%$ & $6.3 \%$ & $115.4 \%$ & $4.8 \%$ & $152.6 \%$ \\
\hline 12 & $12.0 \%$ & $183.7 \%$ & $10.0 \%$ & $118.0 \%$ & $8.0 \%$ & $153.9 \%$ \\
\hline 9 & $10.4 \%$ & $183.5 \%$ & $8.4 \%$ & $116.6 \%$ & $6.7 \%$ & $153.6 \%$ \\
\hline
\end{tabular}

Fig. 3 Cross-sectional SAR maps for the full model and clustered $k=12$ model. The slice shown includes the voxel with the maximum difference in $\mathrm{SAR}_{\text {raw }}$ maps between full and 12-clustered models (see Table 1). The maximum difference resides on the skin of AustinMan where the arm and torso are in contact
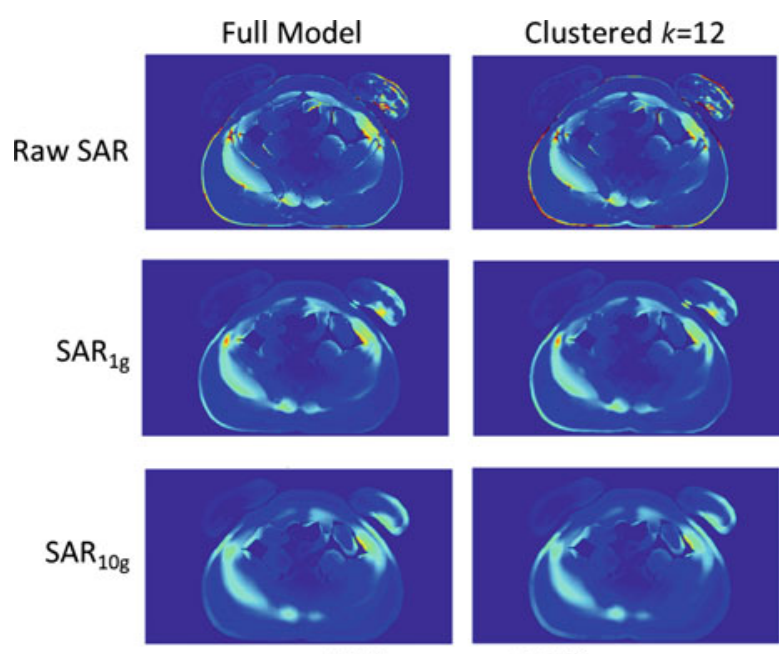

$0 \mathrm{~W} / \mathrm{kg}$

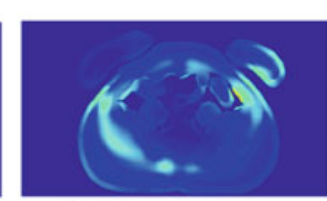

$30 \mathrm{~W} / \mathrm{kg}$

All the results in this study were simulated at the hip bone landmark. The optimal number of clusters may change depending on the imaging landmarks as electric field distribution varies depending on the exposed mass. Another limitation of this study was that only one set of dimensions of RF coil and one field strength were studied. As a previous study showed [11], both can affect the field distribution and the resulting optimal number of clusters.

Our $k$-means clustering approach was only based on dielectric properties. Incorporating the location of the dielectric property may help improve the clustered models. Different approaches such as the Gaussian hidden Markov random field models may be able to help the generation of clustered model based on not only the dielectric properties but also spatial constraints based on neighboring voxels. 
Fig. 4 Scatter plots between full and clustered $\mathrm{SAR}_{1 \mathrm{~g}}$ and $\mathrm{SAR}_{10 \mathrm{~g}}$ values. The linear fit is shown with the lines and $\mathrm{R}^{2}$ values. All of the slope values showed close to 1 . Therefore, there was a one-to-one correlation between each pair. The 33-, 30-, and 15-clustered models showed especially high correlation
Voxel-wise Correlation Between Full and Clustered $S A R_{18}$ and $S A R_{10 g}$ Maps
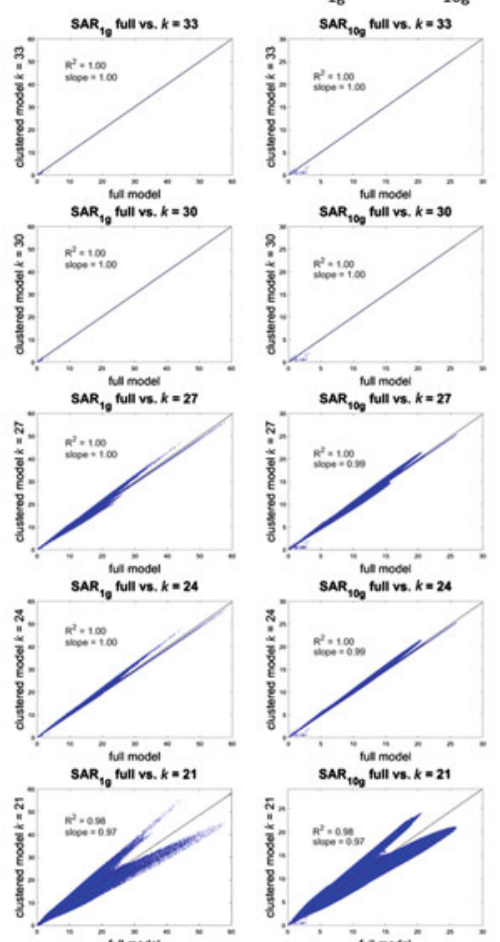

SAR ${ }_{100}$ full vel $k=18$

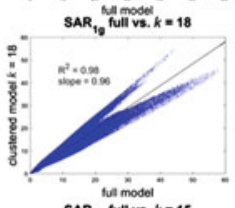

$S_{\text {SAR }}$ full vs. $k=15$
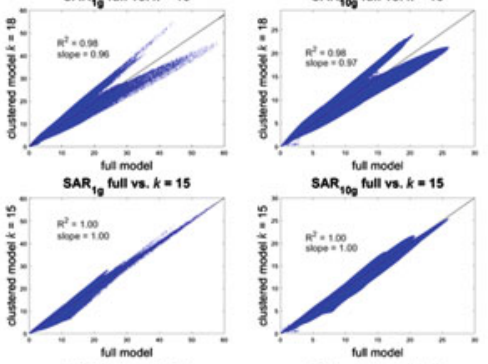

SAR $_{100}$ full vs. $k=15$

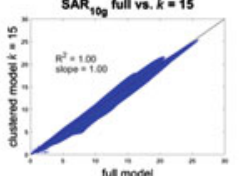

$\mathrm{SAR}_{100}$ full vs. $k=12$
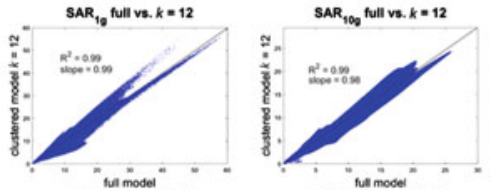

$S_{A R}$ full vs. $k=09$
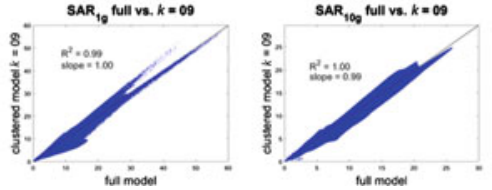


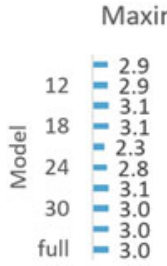

0.0

30.0

60.0

90.0

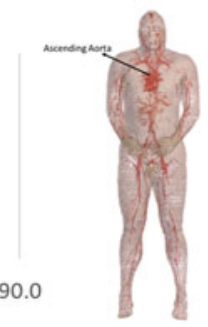

Maximum Etan: Brachial

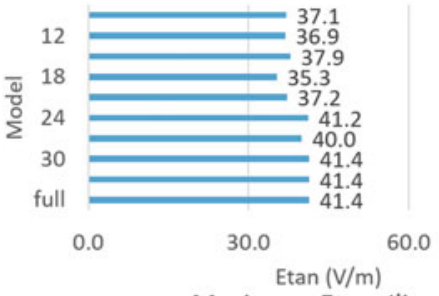

Maximum Etan: lliac

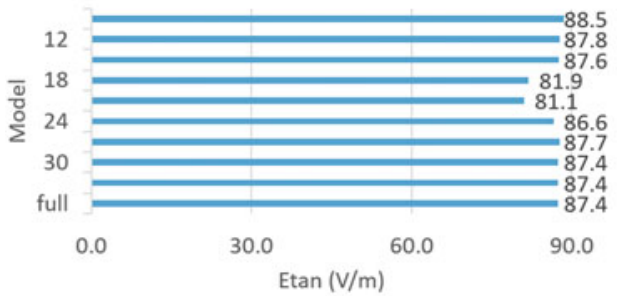

Maximum Etan: Femoral

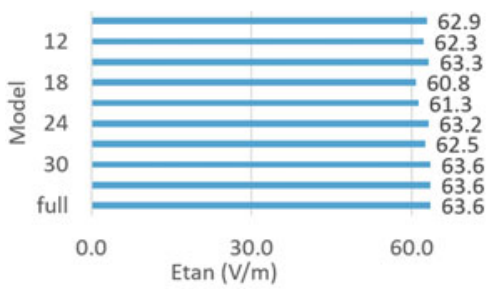

Maximum Etan: Popliteal
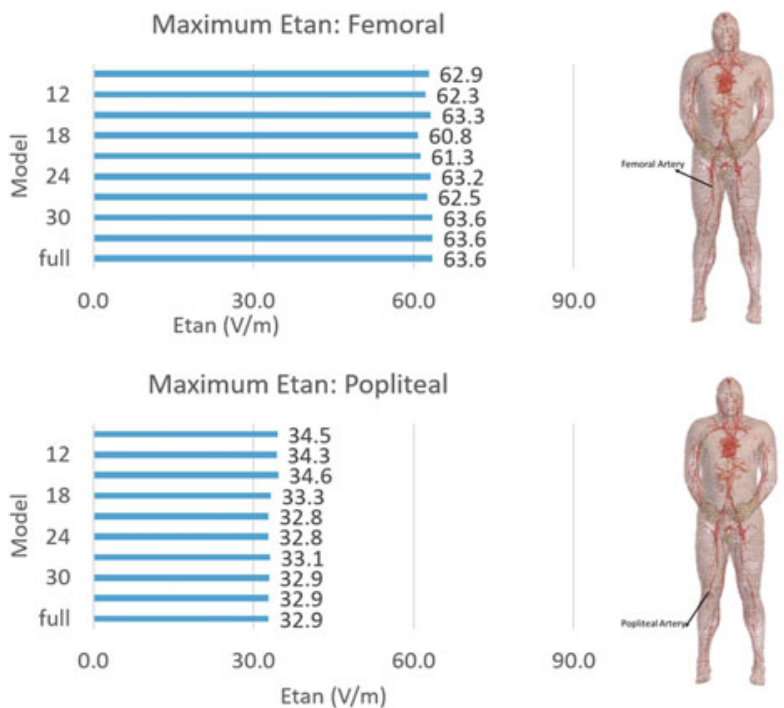

Fig. $5 \mathrm{E}_{\mathrm{tan}}$ on selected blood vessel paths where short stents are commonly implanted are calculated 


\section{Conclusion}

Simplified numerical models based on dielectric properties can show equivalent SAR result. Further investigation of the clustering method may enable efficient MRI safety assessment by simplifying the model generation and reducing computational time.

Acknowledgments This work was supported by the FDA Office of Women's Health and the Research Participation Program at the Center for Devices and Radiological Health administered by the Oak Ridge Institute for Science and Education through an interagency agreement between the US Department of Energy and the US Food and Drug Administration. The authors would like to thank Tayeb A. Zaidi, Trent V. Robertson, Drs. Brian B. Beard, David A. Soltysik, and Eriko S. Yoshimaru for their helpful comments and discussion.

Disclaimer The mention of commercial products, their sources, or their use in connection with material reported herein is not to be construed as either an actual or implied endorsement of such products by the Department of Health and Human Services.

\section{References}

1. Murbach, M., Cabot, E., Neufeld, E., et al. (2010). Local SAR enhancements in anatomically correct children and adult models as a function of position within 1.5 T MR body coil. Progress in Biophysics and Molecular Biology, 107(3), 428-433.

2. Christ, A., Kainz, W., Hahn, E. G., et al. (2010). The virtual family-Development of surfacebased anatomical models of two adults and two children for dosimetric simulations. Physics in Medicine and Biology, 55, N23-N38.

3. Nagaoka, T., Watanabe, S., Sakurai, K., et al. (2004). Development of realistic high-resolution whole-body voxel models of Japanese adult males and females of average height and weight, and application of models to radio-frequency electromagnetic-field dosimetry. Physics in Medicine and Biology, 49, 1-15.

4. Visible Human Project.: https://www.nlm.nih.gov/research/visible/visible_human.html.

5. Massey, J. W., \& Yilmaz, A. E. (2016). AustinMan and AustinWoman: High-Fidelity, anatomical voxel models developed from the VHP color images. Conference Proceedings: Annual International Conference of the IEEE Engineering in Medicine and Biology Society, 3346-3349.

6. Homann, H., Börnert, P., Eggers, H., et al. (2011). Toward individualized SAR models and in vivo validation. Magnetic Resonance in Medicine, 66(6), 1767-1776.

7. Fujimoto, K., Angelone, L. M., \& Lucano, E. et al. (2017) Conf. Proc. BMES/FDA.

8. Benkler, S., Chavannes, N., \& Kuster, N. (2009). Novel FDTD Huygens source enables highly complex simulation scenarios on ordinary PCs. Conf Proc IEEE APSURSI, 2009, 1-4.

9. IEC 60601-2-33. (2010). Magnetic resonance equipment for medical diagnosis testing. 
10. Fujimoto, K., Angelone, M. L., Lucano, E., et al. (2018). Radio-frequency safety assessment of stents in blood vessels during magnetic resonance imaging. Frontiers in Physiology, 9, 1439.

11. Fujimoto, K, Angelone, L. M., \& Lucano, E., et al. (2017). Effect of simulation settings on local specific absorption rate (SAR) in different anatomical structures. In Proceedings of ISMRM Workshop on Ensuring RF Safety in MRI: Current Practices \& Future Directions.

Open Access This chapter is licensed under the terms of the Creative Commons Attribution 4.0 International License (http://creativecommons.org/licenses/by/4.0/), which permits use, sharing, adaptation, distribution and reproduction in any medium or format, as long as you give appropriate credit to the original author(s) and the source, provide a link to the Creative Commons license and indicate if changes were made.

The images or other third party material in this chapter are included in the chapter's Creative Commons license, unless indicated otherwise in a credit line to the material. If material is not included in the chapter's Creative Commons license and your intended use is not permitted by statutory regulation or exceeds the permitted use, you will need to obtain permission directly from the copyright holder.

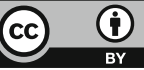

